\title{
Automatic Detection of a Heart ROI in Perfusion MRI Images
}

\author{
Lennart Tautz ${ }^{1}$, Ola Friman ${ }^{1}$, Anja Hennemuth ${ }^{1}$, Achim Seeger ${ }^{2}$, \\ Heinz-Otto Peitgen ${ }^{1}$ \\ ${ }^{1}$ Fraunhofer MEVIS, Bremen \\ ${ }^{2}$ Eberhard Karls University, Tübingen \\ lennart.tautz@mevis.fraunhofer.de
}

\begin{abstract}
This work presents an automatic and robust approach to detect the heart region in MRI perfusion images. Intensity variance is used to identify high variability regions, which are in turn filtered with features based on their shape and position. The approach is validated using 44 clinical data sets against a ground truth generated by experts.
\end{abstract}

\section{Introduction}

Coronary heart disease is a major cause of death, making the detection of functional disorders of the heart muscle (myocardium) a task of high clinical value. Myocardial perfusion can be measured with MR imaging. To this end, an image sequence is acquired with high temporal resolution showing the pass of an administered contrast agent through vessels and myocardial tissue (Fig. 1). The images encompass a region substantially larger than the heart region to be analyzed. Image processing steps such as histogram analysis or registration can be disturbed by unrelated information from areas outside the heart. If a region of interest (ROI) containing only the heart is available, further processing steps can be restricted to the relevant regions and performed in shorter time. We propose an automatic approach that combines knowledge about the anatomy and the acquired image to detect the heart ROI.

Related approaches exist for the detection of the heart in cine MRI sequences. In recent work [1], the use of intensity variance over time to construct a ROI in cine MRI sequences is proposed. The presented approach does, however, assume that RV and LV can be unambiguously identified in the variance image, which
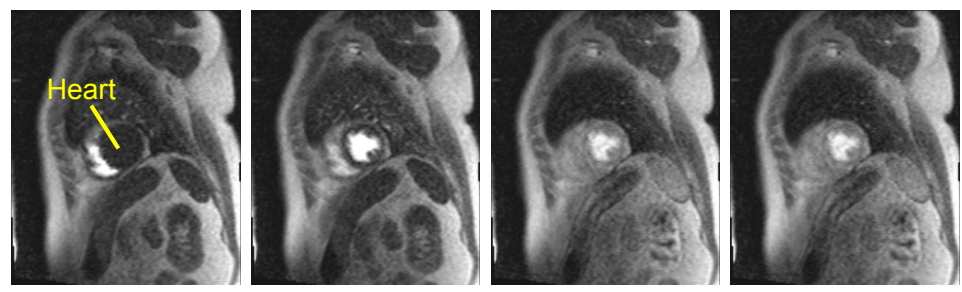

Fig. 1. Time points 4, 11, 19, and 25 of a perfusion MRI sequence showing the pass of contrast agent. 
does not take into account that in contrast-enhanced images other structures, such as the kidney, vessels or artifacts, can show similar variability. We propose a similar approach that is more robust with respect to disturbances from artifacts and other contrast agent-influenced structures. The authors of [2] create a motion map based on intensity differences, which is sensitive to high variability. An alternative approach that uses FFT to characterize intensity variability and to delineate cardiac structures in 4D MRI images is presented in [3]. Spreeuwers et al. [4] search for local maxima in space and time to detect left and right ventricle (LV and RV) in perfusion MRI sequences, but the authors do not explain how they assure that the ROI contains the heart at all timepoints.

\section{Materials and Methods}

\subsection{Data}

For testing, 44 perfusion data sets are used. The data have three to four shortaxis slices with about $8 \mathrm{~mm}$ thickness, an in-plane-resolution of 1.6 to $2.1 \mathrm{~mm}^{2}$, and a gap of up to $24 \mathrm{~mm}$. The images were acquired with a an ECG-triggered SSFP sequence. Each time sequence consists of 40 images, with an sampling interval of about $1 \mathrm{~s}$. Because of the large gap between slices, the data can be processed as a set of several $2 \mathrm{D}+$ time slices. The geometric connection between the slices is in this work exploited in a second step to ensure consistency.

\subsection{Processing pipeline rationale}

The ROI detection is based on the temporal behavior of image values. Cardiac motion and contrast-enhanced blood flow will produce high variability of intensity values over time inside the heart region. In addition to the ventricles, the kidney and other vessels can be present in the image and will exhibit similar intensity curves over time. Because the LV consistently produces a round region in the variance image, it allows for a more robust heart detection than the RV. Comparing LV-specific features for candidate areas extracted by a connected component analysis, non-LV areas can be discarded. This ROI containes only LV and RV areas and determines a bounding box around the heart for the whole sequence. Steps 1 and 2 of the pipeline are executed for each slice of the $2 \mathrm{D}+$ time data set, and steps 3 and 4 merge the preceding results.

Step 1: Temporal Variance. The standard deviation of the intensity over time is calculated (Fig. 2b). The result is thresholded with the value at the 97percentile, retaining only regions with very high variability (Fig. 2c). To remove small holes and false-positives caused by noise, a $3 \times 3$ median filter is applied.

Step 2: Candidate Regions. The candidates for the LV region are then extracted by a connected components analysis. A lower boundary for the component volume of $3 \mathrm{ml}$ has been to empirically determined to exclude remaining 
small regions and vessels outside the heart. Usually, three to five high variability regions will remain, including the LV region, up to two RV regions due to the size and flow behavior of the right ventricle, the kidney and smaller vessels (Fig. 2d). For each of these candidates, two features are calculated: the similarity to an ellipse fitting in the candidate's bounding box, and the distance of the candidate to the image center. The similarity to an ellipse is defined as the Jaccard index $J$ between the area $A_{\text {ellipse }}$ of the ellipse and the area $A_{\text {candidate }}$ of the convex hull of the candidate

$$
J\left(A_{\text {ellipse }}, A_{\text {candidate }}\right)=\frac{\left|A_{\text {ellipse }} \cap A_{\text {candidate }}\right|}{\left|A_{\text {ellipse }} \cup A_{\text {candidate }}\right|}
$$

If the aspect ratio of the candidate is below 0.5 , the candidate is discarded. The distance to center is defined as the inverse scaled distance between the image center and the candidate's center. The distance is inversely scaled to a value between 0 and 1 , where 1 is the minimal distance, and 0 is the maximal distance between the image center and one of the image corners. These two feature scores are added together, and the candidate with the maximal score is selected as the LV region for the current slice (Fig. 2e). A bounding box is computed around this region and extended by $30 \mathrm{~mm}$ on each side to reflect the fact that

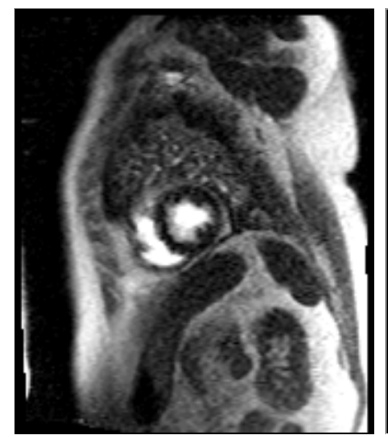

(a) Input image

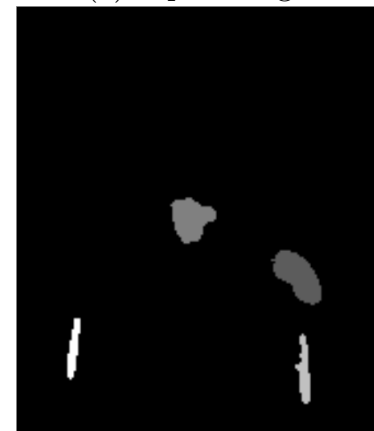

(d) LV candidates

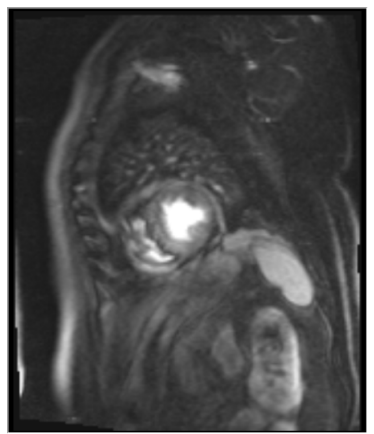

(b) Std. Dev. image

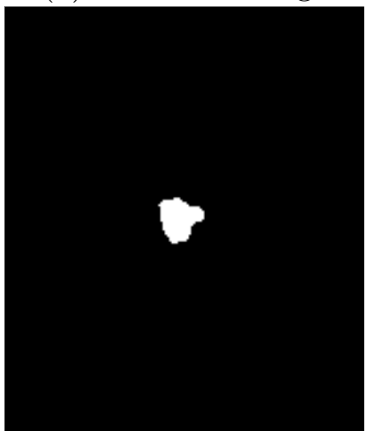

(e) Best LV candidate

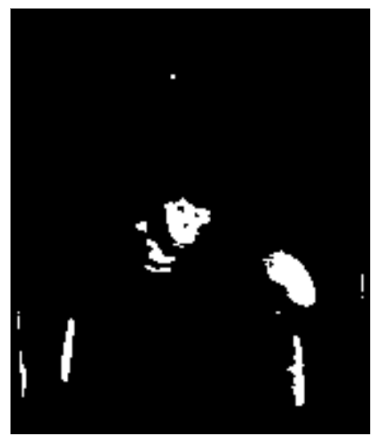

(c) High std. dev. regions

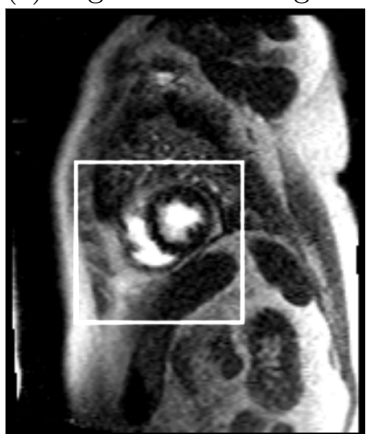

(f) Final result

Fig. 2. Processing pipeling. 
the candidate ROI represents the LV bloodpool without the myocardium. To include the myocardium and a small safety margin, the bounding box must be extended by the typical myocardium thickness.

Step 3: 3D Consistency. When the LV regions have been determined for all 2D slices in the data set, a 3D consistency check is carried out. On single slices, a different region, typically located in the kidney, can obtain the highest score. To remove such outliers, the positions of all regions are compared. Because the true LV regions are assumed to be centered around the long axis, the distance between regions must not exceed $40 \mathrm{~mm}$, allowing for movement and errors introduced by the variability analysis. If a region lies farther away, a new region is selected for this slice. This is repeated until a valid region is found.

Step 4: Bounding box. A bounding box that encompasses the LV in all slices is obtained by calculating the union of the slice bounding boxes. Because this box is determined mainly by information from the center of the LV region, it will not be large enough to contain the moving LV over time. The RV region will usually not be connected to the LV region, so that the box will not cover the heart fully. This is compensated for by stretching the bounding box by a factor of 0.75 into the direction of the RV. The stretching factor reflects a simplified diameter relation of $\mathrm{LV}$ and RV. The relative position of the RV can be inferred from the image orientation, assuming standard heart anatomy (Fig. 2f).

\section{Results}

The algorithm was applied to find the heart ROI in all test data sets. Examples are shown in Fig. 3. The detection takes less than one second per data set on a standard PC with a $3 \mathrm{GHz} \mathrm{CPU}$ and 8 GB memory.

The calculated ROIs were quantitatively evaluated against ground truth ROIs determined manually by three experts. For each data set, the average of the manually drawn ROIs was taken as ground truth and compared with the automatically identified ROI. The automatic ROI was deemed correct if the manual ROI was fully contained by it. If it was not, the maximum difference between the ROI borders was calculated. In 40 of 44 data sets, the ROI was calculated correctly by our approach. In the remaining four data sets, the maximum distance between the manual ROI and the automatic ROI was $6 \mathrm{~mm}$, with a mean of $4 \mathrm{~mm}$. Examples of bounding boxes are shown in Fig. 3.

\section{Discussion}

Our approach provides an automatic and robust method to determine a ROI of the heart in cardiac perfusion MRI images. The ROI detection forms a preprocessing step for further analysis, for instance segmentation of the myocardium and motion correction in the time sequence. Because the detection relies on the 
Fig. 3. Example results (solid: reference ROI, dashed: automatic ROI) (a)-(d) correct ROIs. (e)-(f) failed ROIs.

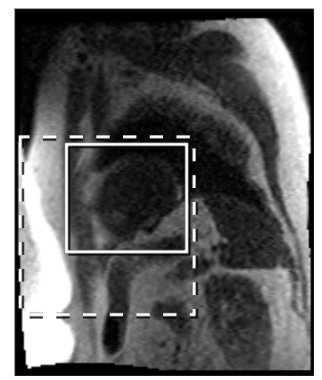

(a) Correct ROI

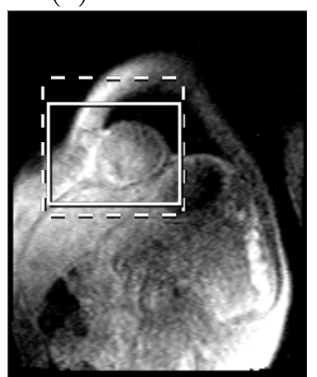

(d) Correct ROI

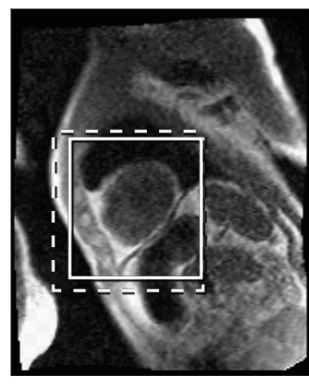

(b) Correct ROI

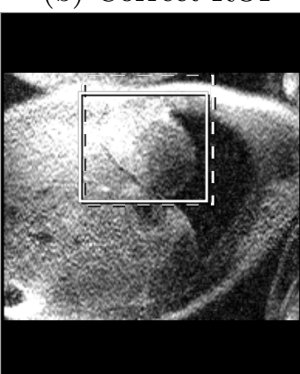

(e) Failed ROI

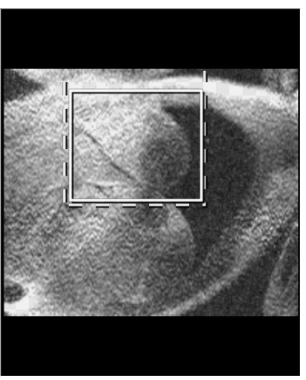

(c) Correct ROI

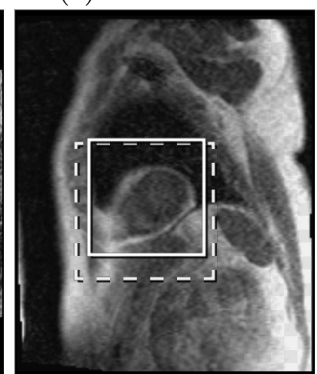

(f) Failed ROI

detection of the LV, perfusion data of hearts with congenital defects can be processed with this method as well.

Because the ROI can be rather large in comparison to the true heart region due to conservative expansion, future work will focus on downsizing overly large ROIs. This will require a detection of the RV region among the region candidates and a detection of the borders in the heart region. A combination of this information will allow the method to calculate a tighter bounding box without sacrificing correctness at critical points.

\section{References}

1. Zambal S, Schöllhuber A, Bühler K, et al. fast and robust localization of the heart in cardiac MRI series. Proc VISAPP. 2008;1:341-6.

2. Pednekar A, Kurkure U, Muthupillai R, et al. Automated left ventricular segmentation in cardiac MRI. IEEE Trans Biomed Eng. 2006;53(7):1425-8.

3. Lin X, Cowan B, Young A. Automated detection of the left ventricle from 4D MR images: validation using large clinical datasets. Adv Image Video Technol. 2006; p. $218-27$.

4. Spreeuwers L, Breeuwer M. Automatic detection of myocardial boundaries in MR cardio perfusion images. Med Image Comput Comput Assist Intervent. 2001; p. 1228-31. 